\title{
Interfacing with adaptive systems
}

\author{
René Ahn, Emilia Barakova, Loe Feijs, Mathias Funk, Jun Hu, Matthias Rauterberg \\ Designed Intelligence Group, Industrial Design Department, Eindhoven University of Technology, Eindhoven, the Netherlands \\ Email address: \\ r.ahn@tue.nl (R.Ahn), e.barakova@tue.nl (E. Barakova), l.m.g.feijs@tue.nl (L. Feijs),m.funk@tue.nl (M. Funk), j.hu@tue.nl (J. Hu), \\ g.w.m.rauterberg@tue.nl (M. Rauterberg)
}

\section{To cite this article:}

René Ahn, Emilia Barakova, Loe Feijs, Mathias Funk, Jun Hu, Matthias Rauterberg. Interfacing with Adaptive Systems. Automation, Control and Intelligent Systems. Vol. 2, No. 4, 2014, pp. 53-61. doi: 10.11648/j.acis.20140204.12

\begin{abstract}
We cast a design view on the interactions that occur when humans face (interconnected) adaptive systems. As humans are also adaptive, the combined behavior of such systems and humans can exhibit various phenomena that are especially of concern to designers of adaptive systems dealing with the inherent complexity of systems, systems' interfaces, interconnectivity, and other design factors. Based on examples of interactions between humans and systems at different levels of complexity, we propose a hierarchical taxonomy of increasingly complex challenges that system engineers will encounter when designing adaptive systems. Among adaptive systems, the taxonomy distinguishes closed and open systems, embodying processes that are unaware or aware, and finally, friendly and hostile. This taxonomy can be of use in designing these systems and their interfaces, as it helps to categorize the information needs of users. In fact, systems at various levels in the hierarchy need to offer certain cognitive affordances for users to operate these systems successfully. We illustrate how complex the information needs of users in these different situations can be, and formulate emerging design research questions. These could be of particular interest to designers who create intelligent systems, products, and related services in a societal context.
\end{abstract}

Keywords: Adaptive Systems, Human Behavior, Interfaces, Systems Design, Taxonomy

\section{Introduction}

Scientific and technological progress enables us to create new types of products, systems, and services. In particular, miniaturization, affordability, and the advancement in design of interactive and intelligent systems extends the range of situations and applications where we can now create products or systems that can react on changes in their environment without explicit prompting from their direct users. These systems often have a repertoire of actuators and sensors through which they perceive certain characteristics of their environment, and a 'policy' which makes them 'choose' an 'appropriate' action adapted to a given situation.

From a user point of view, these systems act independently, adapting to changes in the environment or in the situation, e.g., for traffic situations as will be described below. When such systems are introduced in a given environment, they may bring positive changes and even save users time and trouble. But they also will introduce changes in this environment, changes to which the user needs to adapt, for instance, uncertainty, ambiguous signals, and behavior that changes and adapts over time. Aarts and de Ruyter propose and rank (from low to high adaptivity) the following ambient system intelligence levels: (1) context aware, (2) personalized, (3) adaptive, and (4) anticipatory [1]. Although this specific level system is useful for intelligent environments, we try to address a general niveau by following the definition of complex adaptive systems [2]: A complex adaptive system consists of inhomogeneous, interacting adaptive agents; adaptive means capable of learning; an emergent property of a complex adaptive system is a property of the system as a whole which does not exist at the individual elements (agents) level.

As the number of systems keeps growing, their effect on the environment accumulates, and the user's position in his environment changes, first gradually, but later more extensively [3]. This happens because the user will, eventually, interact more and more with his environment through these systems, so his interaction with his environment becomes more and more indirect and mediated through electronic systems. The range of the environment tends to expand, and the interaction with the environment may become more varied, wide-ranging, 
sophisticated, and extensive, but also more incomprehensible, ambiguous, conditional, complex, and artificial.

We assume that there are clear trends to be observed here, and that designers should investigate these trends closely to achieve a better fundamental understanding of the various trade-offs and design issues that play a role in them. We can see that there are specific problems resulting from these trends, problems that users are currently already facing [4]. Clearly, these problems relate to new design decisions that need a novel framework of thinking which can result from a careful analysis [5].

\section{Interaction Patterns and Societal Trends in Technological Realities}

As humans, we find ourselves involved in complicated causal chains of interaction, where we juggle a large number of external demands and internal objectives. Furthermore, these causal chains are shaped by the distribution of power and the exertion of social pressure. Therefore, this network is seemingly becoming increasingly large, fast, dense, and sophisticated.

Naturally, as social creatures, humans have always been a part of a large network of interactions [6]. Accordingly, our societies have already developed cultural conventions, as well as legal rules that supported our functioning within these networks. However, many of the regulating features that have been developed and which are currently embedded in our habits, institutions, laws and culture, have all evolved under circumstances that may now no longer be warranted. The existing culture, institutions, and laws are still largely based on certain outdated assumptions about the flows of information in the aforementioned causal chains of interaction: Firstly, interaction patterns are still assumed to work at a human pace. This reflects the historical fact that changes in the causal networks surrounding us were largely driven by humans. Secondly, the topology of the causal network was considered to be more or less static or only subject to very slow changes [7]. This reflects the historical fact that the creation of connections in the causal network was costly, labor intensive, and time consuming. Thirdly, our culture still assumes that the timeliness, cost, and frequency of interactions largely depend on proximity, which is indeed still dominant in face-to-face interaction. This reflects the fact that most interactions were direct physical and mechanical interactions, e.g., pushing buttons on the dashboard of a car or operating a screwdriver, and these interactions were therefore clearly localized. Fourthly, another assumption that may have been valid for most civil interactions throughout history, but which is now no longer warranted, is the explicit presence or absence of anonymity, the reciprocity or symmetry (at least in terms of available information) in certain types of interactions between different parties. Finally, our assumptions are still limited to expecting contradictions, ambiguity, and uncertainty in human counterparts of interaction [8]. This is and will be changing drastically: complex intelligent devices, systems of such (remote) devices, and services will involve more uncertainty than ever before [9]. They will operate on more uncertainty, but also create more uncertainty themselves. A good example are automatic stock trading agents, which utilize limited models about the behavior of the market, and by trading, they let the market slowly deviate from the models as they exploit the model with tailored heuristics.

We ought to explain why we think this matters to designers and system engineers [10] [11]. Whereas Industrial Design, until recently, used to be about form giving, efficient manufacturing, product aesthetics, and ergonomics, nowadays it encompasses much more [12]: first, classical products such as coffeemakers and shavers have embedded electronics, so industrial designers also had to take responsibility for the behavior of the embedded software. Then all similar products, and of course also mobile phones and televisions etc., became Internet enabled. So now they are part of complex socio-technical systems and the designers are co-responsible for the efficiency, the trust, and the aesthetics of the interaction with these systems [13]. Consequently, the interactions and considerations addressed in this paper are of utmost importance to the design community [7].

As designers, we now need to acknowledge that many of the basic circumstances that shape human-product interactions are changing rapidly. Our design practice has not yet been adapted to these changes, and we need to make a conscious effort to avoid the threats that these developments may pose and grasp the opportunities that they may offer [14]. To identify these more clearly, we need to thoroughly study how adaptive systems present themselves from a human viewpoint, and how human users deal with such systems, either by operating on the assumptions of causality, or by finding new strategies to cope with the inherent uncertainty of adaptive systems [4].

\section{The Human Point of View in Design}

As the result of actions always lies in the future, purposeful human action is ultimately rooted in expectations [15]. More precisely speaking, what often motivates human action is some change that is (consciously or unconsciously; see [16]) expected as a result of this action. Actions are motivated by the likely changes in the environment that are expected as a result of these actions [17]. The expectations that humans have are dependent on their ideas, reflexes, skills, prejudice, and knowledge as well as on their current assessment of the given situation [18].

Human environments are extremely complex, and stretch far beyond human mental capacities, so humans are never able to fully predict the consequences of their actions [5]. Yet, in many natural situations, in which humans are competent, they are capable of predicting quite adequately how their actions can affect a certain environment. They understand which aspects of an environment tend to remain 
static, and which will change as a result of their direct actions. But that is not all. They are also able to foresee (and deal with) the various spontaneous changes that may arise in the different elements of a given environment. So, as a rule, humans cannot only estimate the potential of their own actions in a given situation, but can also anticipate to a certain extent the spontaneous changes that may happen in various elements of their environment. Humans can have quite detailed expectations about the behavior of their environment, and they may also understand how various independent actors in their environment are interconnected and can influence each other.

For instance, an experienced cook that prepares various dishes simultaneously does not only know that dishes and spoons will remain where he puts them, but he will be able to foresee when to turn the meat or stir the sauce in order to keep it from burning, he knows how to initialize and control the many variables that determine the end result, and may know special tricks that enable him to deal with exceptions: He knows when to add a drop of water to save the mayonnaise. What is crucial for his competence is that he has reliable expectations about the current state of the system 'kitchen' and about the way in which different aspects of the system will evolve, and that he knows where and when to inspect the system to track its development. Last but not least he also knows how to intervene in order to steer the system in the desired direction and to avoid unwanted outcomes.

So, humans are able to predict where spontaneous (i.e. unprovoked) changes in their environment may occur, and how these events can influence the systems the user is currently interacting with. Humans know which elements in the environment they need to (consciously or subconsciously) monitor to manage changes or to react to them. Thus, in the following we will abstract from humans in more theoretical terms as smart adaptive controllers or responders with a limited foresight [19] [20].

To better understand how the human ability to take care of (complex) situations based on limited foresight may be affected (and perhaps even challenged) by some of the trends that we have identified, we will consult a model that can describe a human that 'takes care' of a situation, i.e., a system within an environment. Such a control situation is an environment with actors and products, in which a control task takes place [21]. This definition holds for more traditional 'static' systems as well as for adaptive systems. Such systems are specializations of control situations. The model is inspired by control theory and cybernetics and reframes control theory for 'systems in contexts' (see for 'context' [22]). In this discipline, 'taking care' of a control situation is described as a 'control task'. The following short excursion into cybernetics will help us understand why different control situations lead to different types of control tasks which may be more or less challenging to humans, and it may also help us to identify the designable aspects of a control situation that may make these tasks more or less demanding.

\subsection{Human Control as a Cybernetic Task}

The field of Cybernetics (from the Greek word

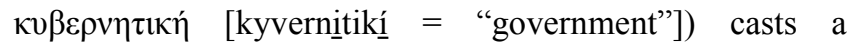
structured view on control tasks [23]. The essence of a control task is that a 'controller' (for instance a human) who performs actions (provides inputs) that keep 'essential variables' of those objects and processes (the system within its environment) that matter to the controller (which may include the controller itself) within desired bounds. The controller protects or shields these variables from the possible influences of external disturbances: "In general, then, an essential feature of the good regulator is that it blocks the flow of variety from disturbances to essential variables" [23, p. 201]. A well-known classical example is the temperature controller in a room: when the (variable) temperature becomes too low, the heater is switched on. When too high, the heater is switched off. When there is a cold wind blowing from outside, that's an external disturbance. In the rest of this paper we focus on one control task chosen for practical reasons: driving a car. This example allows us to illustrate a number of important points of increasing complexity. At the same time we assume most readers are familiar with what it means to drive a car. An example for a user who is interacting with processes involving essential variables that need to be regulated is a person actually driving a car. We can assume that the driving process which involves interactions between the car, the driver, and the environment, is characterized by some state that is changing over time. To drive successfully, the driver needs to undertake many actions, which influence how the state of the process changes over time. These actions, the inputs from the driver (controller) to the process, need to ascertain that essential variables like distances, (relative) velocities, fuel level, engine temperature, or even his own fatigue remain within certain limits.

Such a control process can be modeled as an optimization process. We can assume that the driver tries to minimize some 'error' quantity that can be calculated from the values of certain essential variables. It is important for the driver to keep this error within certain bounds. The driver needs to keep the system state within a 'safe' region. If the driver is not able to do this, an accident may occur.

How the state of the process develops over time does not only depend on the inputs that the controller provides. There will also be external factors that influence the time development of the process. From the viewpoint of the controller these 'external' inputs constitute disturbances over which he has no direct control but for which s/he may have to compensate. In the example of driving a car, external inputs or 'disturbances' can be other drivers on the same road, animals, road conditions, weather conditions, the state of the brakes and the engine, etc. It is important to realize that the state of the process being controlled may not always be completely observable to the controller. Only certain aspects of that process may be observable. The collection of these aspects constitutes a certain (limited) view of the 
controller on the process of interacting in the system.

The human now faces a 'control task'. This task consists of 'steering' the process through the manipulation of the inputs, based on the observations that the view on the process allows. Despite the external disturbances, the controller needs to keep the 'error' of the process within the prescribed bounds. The very simplest example of this notion of being 'within bounds' could be keeping the car on the road. Secondly, respecting the $\min / \max$ speed limits and thirdly keeping a safe distance from obstacles, but other types of bounds, not just literal bounds, are included as well.

The multitude of different influencing factors of a control task needs to be defined structurally. Figure 1 shows a special control situation, namely an adaptive system, consisting of one User A, the process to be controlled, other users (User B and C), and the environment. Embedded in the adaptive system are control tasks, which express the dynamics of the control situation, not just between primary user and process, but also between other users or the environment and the process. Finally, the process state is depicted as being a floating 'dot' that needs to be maintained in a 'safe region' to keep the process and potentially the entire adaptive system alive, by preventing fatal states.

Note that while the human user and the control task are depicted here 'outside' the controlled process, it can and will often be subjected to the consequences of its own control even inside the process. This is definitely the case, for instance, for a user driving a car.

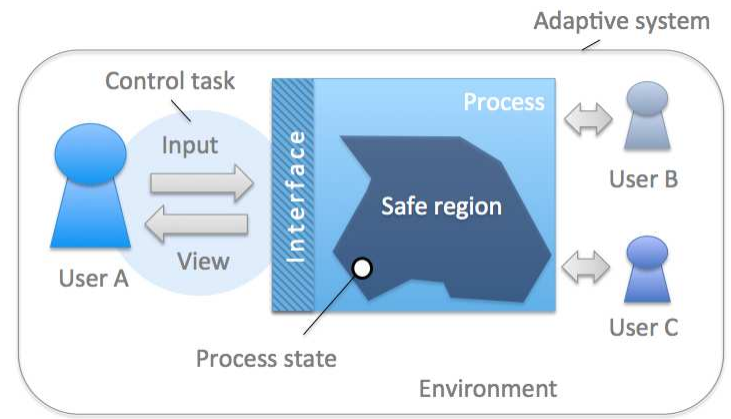

Figure 1. Adaptive system (control situation) involving a control task for a human user: providing the right input for a process via an interface to steer the entire control situation (process and other users within an environment) such that the process state is kept within the safe region.

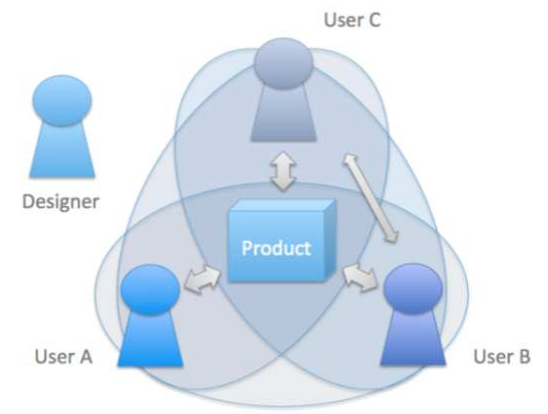

Figure 2. Different points of view on a user-product system in a control situation; the designer determines how User $A$ perceives other communicating Users $B$ and $C$ 'through' the product.

\subsection{A First Person View}

When discussing control tasks related to a specific process we have to take different points of view of different users on the process into account: to one user, for instance, the partially autonomous reactions of the process may appear hostile, or confusing, while another user may experience the process' behavior as appropriate or exactly as expected [24]. Designers of such a process need to adopt the various relevant potential views of key stakeholders or users to design a process that makes sense to them.

In the following, a taxonomy of steering tasks is presented, where a first-person-view from a controller, i.e. a user, to the process and its environment including other users (in total: a control situation) is presupposed as the relevant perspective to classify the steering-task. This presupposition (that tasks are classified as to how they appear from a first-person perspective) is essential, because we want to base design conclusions on our (rather abstract) taxonomy. Figure 2 illustrates this presupposition in the context of a product such as a car in traffic. From the controller's (User A) point of view, not only the physical product is targeted by her steering task, but rather a whole control situation that combines the car (product), the road, signaling, weather (environment), and other users within the same environment. The same holds for all the other users in the control situation respectively. What might be experienced as product behavior can actually be a result of other users' actions rather than truly autonomous behavior of the product.

This is the control situation model, which shall be used in the following to outline important design challenges. The model describes a control situation structurally (cf. Figure 1), but also as a collection of first person views (cf. Figure 2). Understanding an adaptive interaction setting as a connected system of users, process, and environment is a prerequisite of defining the task of designing such a system as a meta-steering task which needs to take all users' steering tasks and experiences (actions and perceptions) into account to create a meaningful and holistic user experience. A designer should be able to temporarily adopt or assume all first person views that can be identified in the system of connected users and products, and design accordingly. Referring to the running example of controlling cars, this would be about how to design a crossing and associated traffic light system that optimizes traffic flow and still prevents accidents as much as possible. The designer has to understand the needs and temptations of various drivers approaching the crossing from different entry points. For innovative products and related processes, this requires the designer to predict and foresee potential future user actions. The taxonomy of steering and control tasks, which we propose in the following, highlights essential distinctions that help to guide the design process.

\section{Taxonomy of User's Control Tasks}

It is clear that control tasks that humans may encounter 
can differ substantially in terms of complexity [25], and difficulty [26]. The control situation model described above helps us to examine and distinguish various relevant factors that may determine how challenging the control task is: apart from the complexity of the system state and its associated dynamics, we also need to consider the nature and extent of the external disturbances, together with the completeness of the view of the controller, and also the tolerances on the errors which are permitted. In this section we will provide arguments to distinguish different types of control tasks. Based on these distinctions we create a taxonomy that can serve as a useful characterization of the main types of control tasks that humans will encounter in future ambient and smart environments. The taxonomy is presented in Figure 3 and its details will be explained below.

When the user perceives an adaptive counterpart (the process), the first distinction we make is between isolated and open systems. This distinction is based on the nature of the external disturbances that may jolt the process, or even the entire system. If the external disturbances to a process are so weak and predictable that their effects can always still be corrected after they have occurred (the 'cared for' process state can always be brought back into the safe region after a disturbance), then it follows that the control task can always be performed based on a view that is limited to the process itself. The entire system can effectively be monitored in isolation, and control can be based on a process view, which is completely unaware of all other events taking place in the environment.

However, if some external disturbances may actually be fatal, in the sense that it cannot be avoided that the process state leaves the safe region (cf. Figure 1) after such a disturbance has occurred, it is necessary to prevent these disturbances from affecting the process in the first place.

For these kinds of systems, the user cannot rely on actions that are solely determined by the view on the process. Once a potentially fatal disturbance has compromised the process, it may be fatally derailed, and it may already be too late to avoid system failure. Instead, the user needs to predict the external disturbance and its effect on the process state. The user's view on the environment of the process is crucial here: He needs to be able to adopt a view that extends beyond the state of the process to be controlled, and he must consider other aspects of the environment, which therefore influence his actions. We can thus formulate the following distinction between isolated and open systems (see Table 1).

To illustrate the distinction between these two different types of control tasks through concrete examples, we look at a user driving and navigating a car through traffic scenario. One of the tasks the user is facing is to keep the car at the desired speed; we can call this cruise control. Cruise control is an isolated control task as it regulates the speed of the car by taking the desired speed given by the driver as the reference value, the current speed as a sensor input and using this input to adapt the speed by accelerating the engine or braking. The task can be executed based on a limited system view, therefore it can, at least in this case, also be easily automated (i.e., modern cars with cruise control).

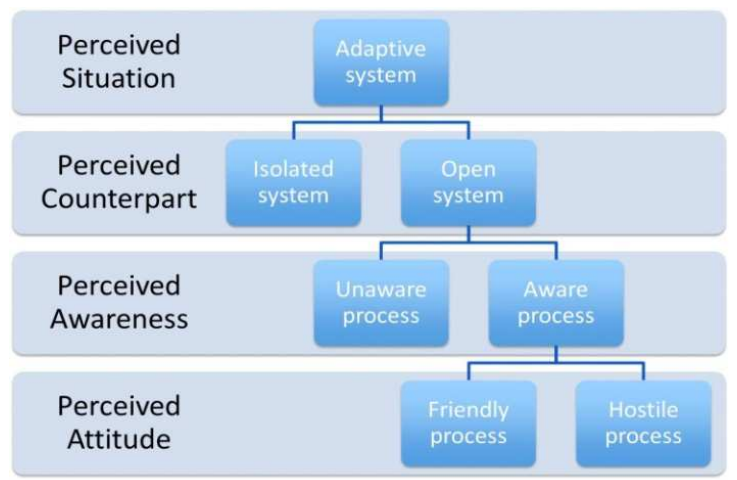

Figure 3. A hierarchical taxonomy of product or process classes is shown with which a user can interact within a control situation. This hierarchy describes the encountered control situations from a first person view of a single user; not shown are other users or the surrounding environment, however, they might be represented indirectly by the product's reactions on their control processes.

But, when navigating in traffic, there can also be external disturbances that are fatal; one needs to avoid collisions with obstacles and other cars. Avoiding the obstacles is a task that cannot be executed from an isolated system view. So, in this case we then deal with 'open' control tasks, where we need to monitor various other aspects of the environment to keep the system on track on the safe region.

Table 1. Distinction between isolated and open systems

\begin{tabular}{llll}
\hline 'Isolated' or 'bounded' system & \multicolumn{2}{c}{ 'Open' system } \\
\hline - & Controllable from isolated & $\bullet$ & Environment view; \\
& system view; & $\bullet$ & Unpredictable behavior; \\
- & Predictable behavior; & $\bullet$ & External disturbances can be \\
- & Disturbances non-fatal; & & fatal; \\
- & Remediation possible; & - & Prevention of disturbances \\
- & Disturbances compensated & & needed, i.e. developments in \\
& after they occurred. & & the system environment need \\
& & to be anticipated and \\
& & preventive action is needed. \\
\hline
\end{tabular}

Table 2. Distinction between unaware and aware disrupting systems (counterparts)

\begin{tabular}{|c|c|}
\hline 'Unaware' Disruptor & 'Aware' Disruptor \\
\hline $\begin{array}{l}\text { Disruptor is blind to user's } \\
\text { input. } \\
\text { Prediction of disruptor's } \\
\text { actions is independent of our } \\
\text { own control actions. }\end{array}$ & $\begin{array}{l}\text { Disruptor is aware of } \\
\text { environment and may even } \\
\text { sense user's input. } \\
\text { Complex prediction, } \\
\text { dependent on our own } \\
\text { action, possibly Intentional } \\
\text { Stance [15]. }\end{array}$ \\
\hline
\end{tabular}

Table 3. Distinction between friendly and hostile (aware and disrupting) systems

\begin{tabular}{|c|c|}
\hline 'Friendly' system & 'Hostile' system \\
\hline $\begin{array}{l}\text { - } \\
\text { pooperative, supportive } \\
\text { - } \quad \text { Signals reliable; } \\
\text { - } \\
\text { System wants to be } \\
\text { predictable. }\end{array}$ & $\begin{array}{l}\text { Challenging, } \\
\text { non-cooperative process; } \\
\text { - } \quad \text { Signals may be unreliable; } \\
\text { System may/want to be } \\
\text { unpredictable. }\end{array}$ \\
\hline
\end{tabular}




\subsection{Dealing with Open Control Tasks}

The tasks dealing with isolated systems as studied by control theory are extensively covered in the literature [27] [28], and are less interesting from a design perspective. What is of interest to designers, are the complexities that can arise in the 'open' control tasks. These are common in real life and do not yield so well to formal analysis. We have seen that, in order to deal with an 'open' control task, a user needs knowledge which extends beyond the immediate process she controls; she needs at least to be able to make some estimations about other elements in the environment that may create disturbances in her task. This presupposed, among others, whether her first person view allows a meaningful decomposition of the environment in various interaction chunks, which is, of course an important design consideration in general [29]. What is crucial here is that the user needs to be able to predict the potential occurrence of certain external disturbances before they do indeed happen. Central questions are:

- What is the effect of her actions (including doing nothing)?

- What is the expected development of other elements in the environment, which may lead to a disturbance and a resulting fatal change of the system state?

If the user finds an element in the environment that may develop into a (fatal) disturbance of the process she may need to take appropriate action beforehand. The user needs to predict the future of elements of the environment that she does not control, in order to influence the process that she needs to control, and she needs to trust her prediction and act accordingly.

The situation resembles somewhat the way modern software engineering copes with unforeseen situations [30]. In early programming languages, the programmer was supposed to specify all situations and code the proper behavior. Contemporary languages support exception handling, a kind of rough classification of unforeseeable situations and what to do if they arise.

How well a user can deal with the presence of a possibly disruptive counterpart, an element of the environment, may now largely depend on the usability, but also reliability, with which she can predict its behavior, and on the complexity of the internal state that is attributed to this disruptor. Her ability to predict depends on her own ability to model this disruptive counterpart. Clearly, the models that she may employ can become very complex, and this is interesting from a design perspective as this model may even be supported by tools. For instance, nowadays, detailed short-term weather predictions are available, that can play an important role as planning aids for other applications. This poses no great problems as long as the behavior of such tools is largely straightforward. This, however, cannot always be guaranteed.

The disruptor that she tries to predict might be itself a process that adapts to the environment, and may produce various reactions. The prediction of such counterpart's behavior is fundamentally more difficult than in isolated systems. In particular, a threshold is crossed if the process to be predicted may somehow sense the users own controlling action and may react to that. In this case, calculating a favorable action depends also on the user's ability to predict how the other process may react to her action, and thus can become a significantly complex task. In such cases, it may even be useful to attribute intentions to the disruptive process, in order to model it.

This leads to the next dichotomy, which distinguishes two types of 'open' control tasks. A user may have to predict the course of another process not under her direct control. This prediction becomes fundamentally more difficult if this process itself is an adaptive process that can take aspects of the surrounding control situation such as the user's own action into account (see Table 2)

We already encountered concrete examples of control tasks involving 'unaware' and 'aware' disruptors: first, reacting to other 'unaware' obstacles in the environment, and second reacting to the other drivers that clearly are 'aware'. Thus, driving a car in an empty road can be seen as a control task with unaware disruptors as the environment such as the road, trees on its side, a nearby forest, and in general the weather are certainly not aware of the driver, but can influence directly the conditions the driver has to deal with: during a thunder storm, a tree struck by lightning might fall onto the road and the rain might reduce visibility, posing an easily life-threatening danger to the driver. Driving in rush hour traffic, on the other hand, forces the driver to deal with 'aware' disruptors.

\subsection{Dealing with 'Aware' Disruptors}

The case where a user needs to respond to an adaptive process that can show various reactions to the user's action is extremely interesting from a design perspective. In this case it is often impossible for the user to predict how this process will develop. On the other hand, however, this problem can now be alleviated because such an adaptive process may signal its planned actions or intentions [31].

The importance of signalling in this case is indisputable: for instance, signalling is often essential for synchronization, as the use of crude signals such as a car's brake lights and turn-signals in traffic already illustrates. Also, signals help to interpret the actions of others and may therefore support cooperation or even social learning [32].

But its design potential reaches much further, as is obvious from importance of phenomena like body language and intonation in cooperation and communication. The use of more intuitive and embodied types of communication through adaptive artefacts therefore seems a promising field of design research: These artefacts may for instance help to direct human attention [33], assist in interaction with autistic children [34], or support inter-human communication in virtual environments [35]. We can conclude that in the interaction between adaptive processes, signalling is extremely important and opens up a huge design space, 
which is still largely unexplored. While humans or adaptive processes may quite successfully communicate with others, the interpretation of their messages is unfortunately not always as reliable as one might hope, even if these messages have been carefully designed. The simple reason for this is that not all processes that we encounter need to be completely friendly or benign, or have interests that are aligned with our own. This means that controllers must, in certain cases, be aware that there may be adaptive processes in their environment that may not have their best interest at heart, and that might try to hinder our success or might even seek to do active harm (see Table 3 ) .

It is clear that this last distinction is quite crucial, and has enormous importance for designers: they can often shape part of a control task, and may, for instance, determine the extent and scope of the signals that are exchanged in a control task. Moreover, for such control tasks, the traffic example is demonstrative as actions of fellow drivers on the road can be both cooperative and uncooperative: cooperative behavior on the road is the usual, expected, predictable case, where accidents are avoided and actually prevented by applying a 'defensive' style of driving, e.g., leaving space, signalling clearly, and avoiding abrupt changes of lanes and directions. On the contrary, there might be bullies, people who drive carelessly or even provoke potentially dangerous situations with unpredictable, irrational moves, i.e. unreliable signals. When messages are not necessarily true, and views are not necessarily reliable, the designer may well be forced to take sides between divergent interests. The person's view on a process clearly plays a decisive role in her success to steer or control that process, and designers can determine that view to a certain extent. Furthermore, in a software and electronics age, the freedom in shaping this view has expanded in many ways. Designers should consider these issues because it may well be that their responsibility has increased lately [36]. It may even be possible, that, in order to take this responsibility, designers may need to cooperate more closely with lawyers, lawmakers, or legal professionals. As designers, we should try to avoid situations where users have to face the challenge of dealing with hostile disruptors.

An 'aware' disruptor is a cyclist who chooses to ignore a red traffic light and cross the road regardless of the traffic regulations (a situation not uncommon in many countries). Although the action is illegal and perhaps immoral, the cyclist still is observing the cars and trying to prevent a sure kill of herself. The car drivers are usually not pleased, but such a cyclist is still a fairly friendly disruptor. An example of a hostile disruptor is someone throwing a stone brick or a tile from a viaduct just to hit a car. Although this is illegal and immoral, it happens nevertheless (fortunately not very often, but it is hard for the police to trace and arrest the brick throwers).

\section{From Theory to Application}

In the last section, a taxonomy of different design challenges has been presented that implies a first-person view on adaptive systems [37]. The challenges that a process seems to pose to a user in a control situation may have a huge impact on the attitude of this user towards her interaction counterpart. As users are often involved in an increasingly more complex network of adaptive systems interfering with multiple users and their environments [38], the designer's challenge is not only to design a part of such a system that behaves according to a single user's expectations, but also to design elements that can function as part of a networked ecology of adaptive systems and still will be perceived by a majority of users as actively supporting tasks under various circumstances in such an environment [39].

While systems traditionally are understood as a composition of functional components and these elements are subordinate to the system, adaptive systems are a dynamic (transient) composition of users (human actors), processes, and the environment, in which interaction is performed as control tasks between the different 'elements' of the adaptive system. One of these elements is the process which users interact with, and, quite naturally, such an element of the ecology will be perceived differently by different users. These differences are not only due to different levels of understanding, expertise, intensity of use, or initial expectations, but first and foremost due to the respective roles of user towards this element. Additionally, these differences are due to the perspective that the different user have on this particular element. Mapping this to the taxonomy view, different users' views can see a particular process in different ways: for instance, a process that seems hostile to one user may seem friendly to another and even 'unaware' to a third. The responsibilities of designers that change the 'fabric' of the network are far from clear. But it seems obvious that they should shoulder some responsibilities: while designers may not be able to avoid that some of their creations will, to some users, appear as hostile disruptors, they might be able to strive towards minimizing these misinterpretations, or perhaps ascertain that their creations, even when perceived this way, will at least communicate via reliable signals.

Certainly, new questions are raised: On the one hand, we need a better understanding how the design can make use of signalling or refinement of the interface between user and process to ensure certain desired perceptions and expectations. On the other hand, we need to research how the design can allow for transparency within the interface and process to show other users' actions and allow them to transparently interact via the process.

\section{Discussion and Conclusion}

It is clear that humans are increasingly entwined in a complex network of (adaptive) systems (which is a complex system in itself) that is rapidly growing and covering many areas of human activity and interest. The speed, size, density and connectivity of this network are increasing rapidly. 
This development offers many unprecedented possibilities to designers, but also poses new questions: We need ways to categorize different positions of humans (or artefacts) in this network, to cater for the various control tasks that humans need to perform, and to be aware of the basic assumptions that we make about these control situations.

In this paper we have proposed a hierarchical taxonomy of user challenges that can act as a starting point to help designers to group control tasks and determine at least some of the basic characteristics of a given control task that users will encounter. The taxonomy may, for instance, suggest in which control situations signaling may play a role, and to what extent controllers can allow themselves to rely on the content of the signals. The taxonomy also indicates that one of the promising areas of design research may well relate to control tasks where the crucial element is the synchronization and cooperation of a human controller with another adaptive controller. While these possibilities are exciting and leave much to be explored, our taxonomy also shows there is a case for concern. Although the characteristics of interactions with friendly or hostile processes are vastly different, it can happen that these types of interactions are extremely difficult to distinguish, both due to the support for long distance connections, and the almost unlimited flexibility and reproducibility of electronic interfaces and underlying processes (cf. challenges noted above).

It is therefore crucial that the control tasks and situations through which humans can interact with other humans or other systems guarantee or at least support interaction with a minimum of fairness. At present it is not at all obvious that this issue is being addressed [40]. If this does not happen, the position of individual humans in the network might become very vulnerable to say the least. If the current trends continue unabated, we have to face and address the following design challenges:

1. Ambiguity: Users will continuously interact (directly or indirectly) with process that can undertake an action they, as a user, did not initiate.

2. Timeliness: These processes may have (and often will have) fast or almost immediate access to information that informs or motivates their actions that users might not have access to.

3. Lack of transparency: Our knowledge about these processes, their existence, their presence, their structure, their true intent, their origin, and their functioning may be nil (or rather limited), even while we are interacting with them.

4. Control: Through these processes, we may participate in control situations, i.e. interacting with adaptive systems or other human beings, in ways that are not primarily determined by our intentions or attitudes towards these humans, but which are largely shaped by the way our interfaces to them are structured and organized.

It is fair to say that these trends are worrying. It is therefore imperative for us as designers and system engineers that we support and advocate the design of systems and the adoption of rules and practices that strengthen the position of individual humans in such adaptive environments.

\section{Acknowledgements}

This paper is the result of the discussions about 'intelligence', 'adaptivity' and other related topics among the authors and with Karl Tuyls and Razvan Cristescu. We are grateful for their valuable contributions.

\section{References}

[1] E. Aarts and B. de Ruyter, "New research perspectives on Ambient Intelligence," Journal of Ambient Intelligence and Smart Environments, vol. 1, pp. 5-14, 2009.

[2] J. H. Holland, "Complex adaptive systems," Daedalus, vol. 121, pp. 17-30, 1992.

[3] K. Kelly, Out of Control: The Rise of Neo-biological Civilization, Reading, Mass.: Addison-Wesley, 1994.

[4] D. Benyon, "Adaptive systems: A solution to usability problems," User Modeling and User-Adapted Interaction, vol. 3, pp. 65-87, 1993.

[5] H. Hagras, V. Callaghan, M. Colley, G. Clarke, A. Pounds-Cornish, and H. Duman, "Creating an ambient-intelligence environment using embedded agents," Intelligent Systems, IEEE, vol. 19, pp. 12-20, 2004.

[6] B. Latour, "On actor-network theory: A few clarifications plus more than a few complications," Soziale Welt, vol. 47, pp. 369-381, 1996.

[7] S. Evenson, J. Rheinfrank, and H. Dubberly, "Ability-centered design: From static to adaptive worlds," interactions, vol. 17, pp. 75-79, 2010.

[8] T. Rakow and B. R. Newell, "Degrees of uncertainty: An overview and framework for future research on experience-based choice," Journal of Behavioral Decision Making, vol. 23, pp. 1-14, 2010.

[9] E. Lopes, Decision-Making under Uncertainty and Complexity: A Grounded Theory Approach, Saarbrücken: VDM Verlag Dr. Müller, 2010.

[10] C. Hummels and J. Frens, "Designing disruptive innovative systems, products and services: RTD process," in Industrial Design-New Frontiers, D. Coelho, Ed., Shanghai: Intech Open Access Publisher, pp. 147-172, 2011.

[11] K.-H. Huang and Y.-S. Deng, "Social interaction design in cultural context: A case study of a traditional social activity," International Journal of Design, vol. 2, pp. 81-96, 2008.

[12] S. Kyffin and P. Gardien, "Navigating the innovation matrix: An approach to design-led innovation," International Journal of Design, vol. 3, pp. 57-69, 2009.

[13] S. A. Rijsdijk, E. J. Hultink, and A. Diamantopoulos, "Product intelligence: Its conceptualization, measurement and impact on consumer satisfaction," Journal of the Academy of Marketing Science, vol. 35, pp. 340-356, 2007.

[14] M. Davis, "Why do we need doctoral study in design?," International Journal of Design, vol. 2, pp. 71-79, 2008. 
[15] D. C. Dennett, The Intentional Stance, MIT Press: A Bradford Book, 1989.

[16] F. Acker, "New findings on unconscious versus conscious thought in decision making: Additional empirical data and meta-analysis," Judgment and Decision Making, vol. 3, pp. 292-303, 2008.

[17] P. Dourish, Where the Action is: The Foundations of Embodied Interaction, MIT Press: Bradford Books, 2004.

[18] A. Flammer, Erfahrung der eigenen Wirksamkeit: Einführung in die Psychologie der Kontrollmeinung, Bern: Huber, 1990.

[19] A. Clark, Being There: Putting Brain, Body, and World Together Again, Cambridge, Mass.: MIT Press, 1997.

[20] B. Schmidt, "Human factors in complex systems: The modelling of human behaviour," in Proceedings 19th European Conference on Modelling and Simulation, Riga, Latvia, pp. 1-10 (CD), 2005.

[21] Z. Gao, "Active disturbance rejection control: A paradigm shift in feedback control system design," in Proceedings of the 2006 American Control Conference, Minneapolis, Minnesota, USA, June 14-16, pp. 2399-2405, 2006.

[22] P. Dourish, "What we talk about when we talk about context," Personal and Ubiquitous Computing, vol. 8, pp. 19-30, 2004.

[23] R. W. Ashby, An Introduction to Cybernetics, 2nd ed., London, UK: Chapman and Hall, 1957.

[24] P. Houston, M. Floyd, and S. Carnicero, Spy the Lie: How to Spot Deception the CIA Way, London: Icon Books, 2012.

[25] K. Byström and K. Järvelin, "Task complexity affects information seeking and use," Information Processing \& Management, vol. 31, pp. 191-213, 1995.

[26] P. Robinson, "Task complexity, task difficulty, and task production: Exploring interactions in a componential framework," Applied Linguistics, vol. 22, pp. 27-57, 2001.

[27] R. E. Kalman, "A new approach to linear filtering and prediction problems," Journal of Fluids Engineering, vol. 82, pp. 35-45, 1960.

[28] T. Sugie and T. Ono, "An iterative learning control law for dynamical systems," Automatica, vol. 27, pp. 729-732, 1991.

[29] S. Wensveen, T. Djajadiningrat, and K. Overbeeke, "Interaction frogger: A design framework to couple action and function through feedback and feedforward," in
Proceedings of the 5th conference on Designing Interactive Systems: Processes, Practices, Methods, and Techniques, Cambridge, MA, USA, pp. 177-184, 2004.

[30] K. Schwaber, Agile Project Management with Scrum, vol. 7, Redmond: Microsoft Press, 2004.

[31] J. Redish, "Expanding usability testing to evaluate complex systems," Journal of Usability Studies, vol. 2, pp. 102-111, 2007.

[32] N. E. Miller and J. Dollard, Social Learning and Imitation, New Haven, CT, US: Yale University Press, 1941.

[33] E. Deckers, S. Wensveen, R. Ahn, and K. Overbeeke, "Designing for perceptual crossing to improve user involvement," in Proceedings of the ACM SIGCHI Conference on Human Factors in Computing Systems, Vancouver, BC, Canada, pp. 1929-1938, 2011.

[34] E. Barakova, J. Gillessen, and L. Feijs, "Social training of autistic children with interactive intelligent agents," Journal of Integrative Neuroscience, vol. 08, pp. 23-34, 2009.

[35] J. Hu and L. Feijs, "A distributed multi-agent architecture in simulation based medical training," in Transactions on Edutainment III. vol. LNCS 5940, Z. Pan, A. Cheok, W. Müller, and M. Chang, Eds., Berlin Heidelberg: Springer, pp. 105-115, 2009.

[36] T. Swierstra and K. Waelbers, "Designing a good life: A matrix for the technological mediation of morality," Science and Engineering Ethics, vol. 18, pp. 157-72, 2012.

[37] G. Bateson and M. Mead, "For god's sake, Margaret," Convolution Quarterly, vol. 10, pp. 32-44, 1976.

[38] D. Preuveneers, J. Van den Bergh, D. Wagelaar, A. Georges, P. Rigole, T. Clerckx, et al., "Towards an extensible context ontology for ambient intelligence," in Ambient Intelligence. vol. 3295 (LNCS), P. Markopoulos, B. Eggen, E. Aarts, and J. L. Crowley, Eds., Berlin Heidelberg: Springer, pp. 148-159, 2004.

[39] R. Holzer, H. de Meer, and C. Bettstetter, "On autonomy and emergence in self-organizing systems," in IWSOS 2008. vol. LNCS 5343, K. A. Humme and J. P. G. Sterbenz, Eds., Berlin Heidelberg: Springer, pp. 157-169, 2008.

[40] R. Gorbunov, E. Barakova, and M. Rauterberg, "Design of social agents foundations on natural and artificial computation," in Foundations on Natural and Artificial Computation. vol. 6686 (LNCS), J. Ferrández, J. Álvarez Sánchez, F. de la Paz, and F. Toledo, Eds., Berlin Heidelberg: Springer, pp. 192-201, 2011. 